\title{
Modification of an Automated Liquid- Handling System for Reagent-Jet, Nanoliter-Level Dispensing
}

BioTechniques 30:878-885 (April 2001)

\author{
J. Steven Hicks, Brent W. \\ Harker, Kenneth L. Beattie, \\ and Mitchel J. Doktycz \\ Oak Ridge National Laboratory, \\ Oak Ridge, TN, USA
}

\section{INTRODUCTION}

For greater economy and higher throughput, biochemical assay volumes are being scaled for use in small-format vessels. This is evidenced by the development and implementation of highdensity microplates. These plates can contain 1536 wells or greater on the same overall dimensions as a standard 96 -well plate $(7,12)$. The overall plate dimensions are retained, allowing for the use of standard plate-handling equipment, while the well volumes are greatly reduced. Also being adapted for screening purposes are microarrays (10, 14). Microarrays, typically of reduced dimensions compared to microplates, are being used for nucleic acid analyses. Applications in genomics, such as for mRNA expression profiling or DNA sequence analysis, are becoming common with new applications on the horizon. These microarrays can be constructed on various materials with sample densities of 100-10000 features $/ \mathrm{cm}^{2}$. Emerging applications, large combinatorial chemical libraries, and genome sequence data are driving highdensity screening formats. However, implementation of these high-density formats is hindered by the lack of robust liquid-handling instruments that operate at such a reduced scale. These high-density formats demand high-precision, sub-microliter liquid handling.

Several approaches and commercial instruments are addressing these new liquid-handling needs (1). For example, various "touch-off" spotting techniques for high-density arraying have been developed. These approaches employ either machined pins or small-diameter tubing to transfer a small volume of reagent $(2,4-6)$. Pin-based approaches transfer reagents by capillary action, while syringe pumps are used to meter reagents when using small-diameter tubing. The delivery of reagents by touch-off methods requires contact between the solution to be dispensed and the target surface. If the droplet preferentially adheres to the dispensing tip, then the sample transfer is prevented. Typically, for pin-based spotting, the volume dispensed is related to the physical dimensions of the dispensing tip and is preset. This approach is becoming commonplace for creating high-density microarrays on surfaces, but it is less suitable for reagent transfer between microplates. An additional restriction with this touch-off approach to reagent delivery becomes apparent when using multiple-dispensing tips. Parallel sample handling greatly increases sample transfer rates; however, addressing the individual tips is not possible because all dispensing tips touch off at the same time. This prevents the reformatting of the sample order and position.

Another approach to small-volume liquid dispensing is found in the mature field of ink-jet printing. Typically, desktop printers use either piezoelectric or thermal actuation mechanisms and can be designed to dispense volumes of a few picoliters (8). The same basic principals used in these printers are beginning to find their way into fluid-handling instruments for biomedical applications $(3,11,13,15,16)$. This alternative approach is not hindered by the shortcomings of pin-based touch-off methods because the droplets are eject- 
Table 1. Summaries of Single Dispensing Event Data Using Fluorescent Assay

\begin{tabular}{|lccccccc|}
\hline $\begin{array}{l}\text { Pulse } \\
\text { Width } \\
(\mu \mathbf{s})\end{array}$ & $\begin{array}{c}\text { Average } \\
\text { Volume } \\
(\mathbf{n L})\end{array}$ & $\begin{array}{c}\text { Low Value } \\
(\mathbf{n L})\end{array}$ & $\begin{array}{c}\text { High Value } \\
(\mathbf{n L})\end{array}$ & $\begin{array}{c}\text { No. of } \\
\text { Measurements }\end{array}$ & SD & $\begin{array}{c}\text { Volume by } \\
\text { Weighing } \\
\text { cv (\%) }\end{array}$ & $\begin{array}{c}\text { Technique }(\mathbf{n L}) \\
500\end{array}$ \\
\hline 8.6 & 6.0 & 10.3 & 35 & 1.0 & $11.7 \%$ & 15.3 \\
1000 & 39.0 & 32.0 & 42.0 & 21 & 2.4 & $6.2 \%$ & 34.5 \\
2000 & 101.0 & 96.0 & 106.0 & 16 & 2.4 & $2.3 \%$ & 92.0 \\
4000 & 213.0 & 201.0 & 228.0 & 9 & 8.5 & $3.9 \%$ & 192.0 \\
\hline
\end{tabular}

ed from the tip. Volume modulation can be accomplished by repeated dispensing events, and the tips are individually addressable. Unfortunately, desktop printers utilize micro-machined print heads that require significant development time and cost. Another approach to fluid jetting employs high-speed solenoid valves. These valves are used primarily for industrial applications (i.e., labeling) and have been adapted for chemical dispensing (9). Reagent jetting is accom plished by rapidly opening and closing the valve while applying fluid pressure to it. Volume modulation is possible by altering the amount of time the valve is open and the flow rate through the valve. This approach to fluid dispensing is relatively straightforward, compared to piezoelectric or thermal-jetting techniques, and is easily adapted for sub-microliter fluid handling.

Here, we describe the modification of a MICROLAB ${ }^{\circledR} 2200$ (Hamilton Company, Reno, NV, USA) for reagent-jet fluid dispensing using highspeed solenoids. Our objective was to take advantage of the flexibility and software interface of a commercial liquid-handling system and enhance it for sub-microliter fluid handling and highdensity arraying. Specific modifications include the incorporation of highspeed solenoid valves, electronics for driving the valves, sapphire-tipped nozzles, and a high-resolution substage. The costs of these components are small compared to the replacement of the instrument with a dedicated array maker, and the modifications could be adapted for other systems or as a standalone system. We routinely use the instrument for the construction of DNA microarrays and are beginning to apply it for the assembly of small-volume biochemical reactions.

\section{MATERIALS AND METHODS}

\section{Basic Fluid-Handling System}

The modifications were performed on a MICROLAB 2200. Our version of this instrument is equipped for multiprobe, low-volume sample handling. This outfits the instrument with a total of eight syringe pumps, fitted with 10or $50-\mu \mathrm{L}$ syringes and narrow bore 22 gauge tubing. The system also uses a Masterflex ${ }^{\circledR}$ peristaltic pump (ColeParmer, Vernon Hills, IL, USA) for high-volume flushing. The pump is controlled by a Intelligent Instrumentation 40-Channel Digital Input/Output (I/O) Board (Burr-Brown, Tucson, AZ, USA), which is installed in a Pentium computer and is used for the control of the ink-jet driver electronics described below. Instrument control is via Eclipse software Version 4 (Hamilton Company). Additional modifications include the addition of a Serial MVP Valve (Hamilton Company). The valve has three ports and contains a $90^{\circ}$ plug. It is inserted between the peristaltic pump and the distribution manifold that feeds the top ports of the syringe pump plug valves. This extra valve functions to switch fluid feed between the peristaltic pump and a pressurized water bottle that provides the pressure for reagent jetting. The bottle is a 4-L solvent bottle housed in a plastic bottle carrier (VWR Scientific, West Chester, PA, USA) and capped with a two-port fittings cap (Millipore, Bedford, MA, USA). The water bottle is plumbed to the MVP valve and to a pressure regulator mounted on a helium gas cylinder.

\section{Ink-Jet Delivery System}

Valves and mounting. The high- speed solenoid valves were purchased from the Lee Company (Westbrook, CT, USA) and were mounted in place of the MICROLAB 2200's dispensing tips. On the outlet side of the valve, a sapphire-tipped nozzle was connected by 28 -gauge tubing (Small Parts, Miami Lakes, FL, USA). The nozzle was obtained from Bird Precision (Waltham, MA, USA) and consisted of a 0.005 -inch $(125 \mu \mathrm{m})$ orifice mounted in a $38-\mathrm{mm}$ long [0.020-inch (0.51 $\mathrm{mm}$ ) inner diameter and 0.035 -inch $(0.89 \mathrm{~mm})$ outer diameter] stainlesssteel tube. The connection between the 28-gauge tubing and the outlet nozzle was accomplished using a short piece (approximately $5 \mathrm{~mm}$ ) of microbore tygon tubing (Small Parts). A custom holder was constructed for mounting the dispensing tips to the MICROLAB 2200's arm. Drawings are available upon request.

Electronic control circuit. The valve-driving circuit is based on the "spike and hold" method suggested by the valve manufacturer. A high-voltage spike of $42 \mathrm{~V}$ for $0.15 \mathrm{~ms}$ is used to open the valve, followed by a hold voltage of $8 \mathrm{~V}$ for the remainder of the time the valve is open. The high-voltage excitation cannot be used to keep the valve open for an extended time because resistive heating would destroy the solenoid windings. A diagram of one channel of the circuit used for driving one ink-jet valve is shown in Figure 1 . In practice, the operator enters the time ( $\mu \mathrm{s})$ for the overall pulse length for each dispensing event. For simplicity, the timing function is executed in software and not in the driving circuit hardware. For other applications, we use a clock crystal and programmable divide-by-n counter circuit for fast-timing functions. The simple 
software timing method is described below in the Software Controls section.

The parts for the circuit were obtained from Digi-Key (Thief River Falls, MN, USA). A custom circuit board containing 16 channels was constructed. This board is mounted inside an $11 \times 11.7 \times 5.5$-inch instrument case (Digi-Key) along with two power supplies. The high-voltage spike is supplied by a 48-VDC linear supply (DigiKey). A 12-VDC linear power supply (Digi-Key) is used to drive two voltage regulators that produce the other voltages needed by the driver board. The 8 -VDC hold voltage is derived from a 1-Amp, 8-V three-terminal voltage regulator. The transistor transistor logic (TTL) 5 VDC is derived from a 1Amp, 5-V, three-terminal voltage regulator. This board is capable of operating up to 16 valves, with each valve having a dedicated enable-logic control line that allows individual control or simultaneous control. A zener diode is used to assist in removing energy from the circuit to allow for the rapid pulsing of the valve. Detailed parts list and board layouts are available from the author.

\section{Substage}

An X-Y motion stage was added to the right-hand side of the deck of the MICROLAB 2200 for higher-resolution movements. The stage is a MAXY4009 Assembled UniSlide ${ }^{\circledR}$ XY Table (Velmex, Bloomfield, NY, USA) with outboard limit switches and stepper motors. The stage uses 9.53$\mathrm{mm}$ lead screws with a $2.5-\mathrm{mm}$ pitch. With half-stepping, a resolution of 6.35 $\mu \mathrm{m}$ is theoretically possible. We used a VP9000 controller to control and drive the stage. The stage is mounted to a 13 $\times 13 \times 0.25$-inch $(33 \times 33 \times 6.4 \mathrm{~mm})$ aluminum plate that is mounted to the deck of the MICROLAB 2200 with 8-32 screws and the existing tapped holes. A $9.625 \times 10.75 \times 0.5$-inch $(244$ $\times 273 \times 12.7 \mathrm{~mm}$ ) magnetic stainlesssteel plate is used as the stage for holding the target plates or slides. The stage is milled to produce a raised edge at one

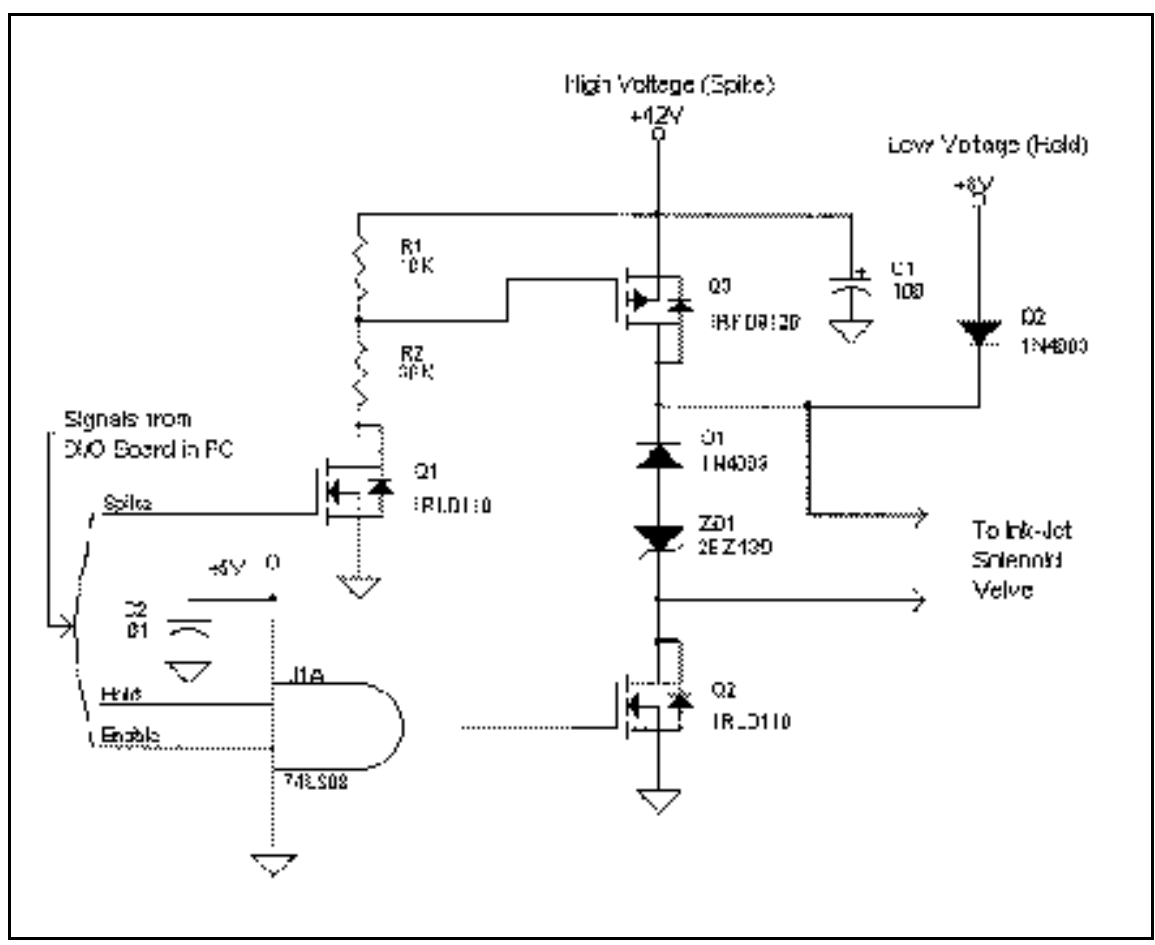

Figure 1. Electronic circuit for driving one solenoid valve. Three lines of one port on the digital I/O card are used to control the operation of the valve driver circuit. The "spike" signal switches on the high voltage, and the "hold" and "enable" signals switch the low side of the ink-jet valve to ground to com plete the circuit. To open the valve, these three signals are sent simultaneously. After a short time, the spike signal is turned off, and the valve is held open with current that flows from the low-voltage supply through D2 and Q2 for the remainder of the pulse width. When the hold or enable signals are turned off, the circuit is opened and the ink-jet valve closes. corner of the plate. This is used for registering and holding the target plates in position with the aid of magnetic strips.

\section{Software Controls}

The standard Eclipse software is used for operating the MICROLAB 2200. This software is extensible through the use of the filecall command. In this way, Eclipse has the ability to call executable files that can perform additional tasks while the instrument is being operated. User-defined information entered into the Eclipse software is communicated to the external software through disk files. This feature was exploited for controlling the ink-jet valves (with high-precision pulse durations) and the $\mathrm{X}-\mathrm{Y}$ substage (i.e., the spacing and number of dispensing events). Because Eclipse is a DOS-based software package, the external software is written and compiled in Microsoft ${ }^{\circledR}$ Quick Basic 4.5 and uses libraries supplied by the manufacturer for controlling the TTL card. The external software is not required for driving the ink-jet valves with coarse timing increments because the TTL command in Eclipse sends signals to the same digital I/O board. The ink-jet valves can be driven directly in Eclipse using this com mand. The TTL command is typically used for the washing and aspirating steps where the valve is held open for long periods of time. The command can also be used for dispensing events; how ever, control of the valve is limited by Eclipse's millisecond timer.

Software loops are used to provide repeatable, sub-millisecond timing delays in the executable program. Typically, two delay loops are used. One is a fixed-loop count for generating a delay of $150 \mu$ s (the high-voltage spike). The other loop is a variable-delay count for generating the total pulse duration, which is entered into the Eclipse program by the operator as the number of microseconds desired. The delay loop is easily calibrated with an oscilloscope for observing the pulse widths versus the loop counts. The timing of the software delay can be modeled with a fixed portion that represents software overhead and a linear portion that scales with loop count. For pulse widths of $150-5000 \mu \mathrm{s}$, this offset (fixed portion) and slope (linear portion) modeling 


\section{Research Report}

works quite well; however, this type of timing is not portable. The softwaregenerated delay will require recalibration when it is used on other computers with different CPU speeds.

There are several approaches to generating accurate timing pulses for firing the ink-jet valves. For this application, we chose to let the software generate the timing delays because we had access to the Burr-Brown digital I/O card that is used by the MICROLAB 2200. Because this card does not have any timer or counter hardware that can be used to directly generate accurate timing pulses, variable delays are generated in the Quick Basic DOS software. This removes the need to add external hardware to the PC to provide this function. As mentioned earlier, later versions of the valve driver circuit use a clock to generate an accurate time reference on the driver circuit board for timing all delivery pulses. This new system operates with Visual Basic and Windows ${ }^{\circledR} 95$ and does not interface with the MICROLAB 2200.
The executable Quick Basic software can also control the substage using the Velmex VP9000 controller that is connected to an RS-232 serial port. The specific commands for stepping the stages and controlling their speeds and accelerations are incorporated into the executable software for seamless motion and dispensing.

\section{Volume Characterization}

The dispensed volumes were characterized by one of two means. Repeated dispensing events were deposited into a pre-weighed vial and weighed on an analytical balance to determine the average delivery volume. Typically, either 500 or 1000 deliveries were added together. No corrections were made for evaporation. The second characterization technique used a $10-\mathrm{mM}$ stock solution of fluorescein prepared in 10 $\mathrm{mM}$ Tris-EDTA buffer, $\mathrm{pH}$ 8.2. This solution was aspirated and delivered into individual wells of a 384-well microplate loaded with $10 \mu \mathrm{L}$ water. The fluorescence values were read using an HTS7000 plus plate reader (Applied Biosystems, Foster City, CA, USA) and compared to a standard dilution of the fluorescein stock.

\section{RESULTS AND DISCUSSION}

Figure 2 displays a photograph of the modified MICROLAB 2200, show ing the layout of the various components and details of the valve and dispensing tip mounting. The solenoidactivated, reagent-jetting system is comprised of the pressure-regulated fluid reservoir, the solenoid valve, and the dispensing tip. The sapphire-dispensing probe is added to allow for precise droplet delivery. Other dispensing tips, such as hypodermic tubing, glass or plastic tips, work suitably; however, the orifice dimensions and the decrease in pressure across the nozzle will affect the dispensed volume. The dispensing probes have a polished orifice of 125 $\mu \mathrm{m}$ and allow for a better release of

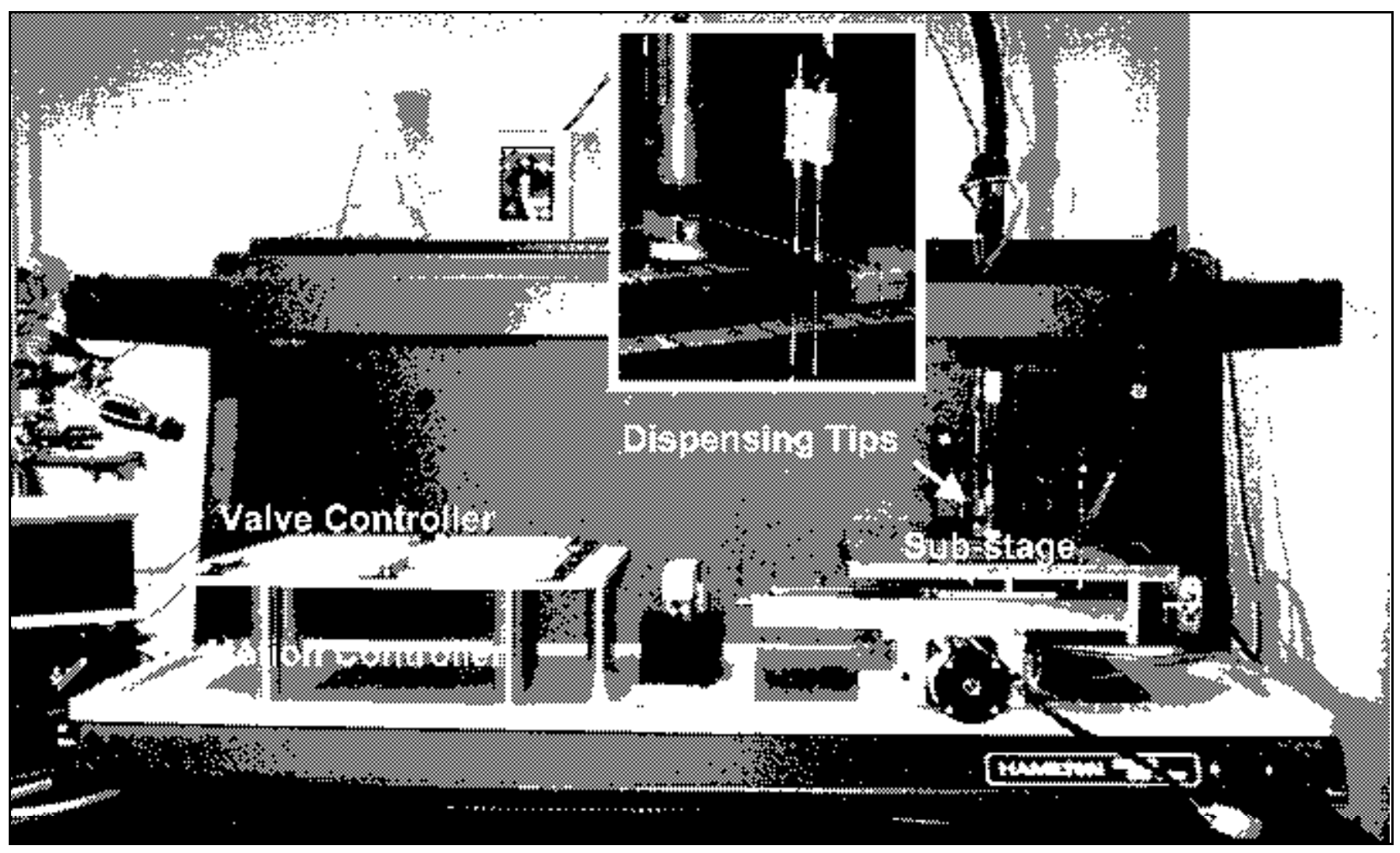

Figure 2. Photo of MICROLAB 2200, modified for reagent jet dispensing. The relative positions of the high-resolution substage, motion controller, and valve driver electronics are shown. The substage is used for the mounting of microscope slides during microarray construction. The inset picture shows the mounting of two dispensing tips and the high-speed solenoid valves. 
droplets compared to hypodermic tubing. Further, these tips are of sufficient length and diameter for accessing most microplates. The overall distance between the valve and the dispensing tip did not appear to adversely affect dispensing for tubing lengths up to 3 inches $(76 \mathrm{~mm})$. Using the 0.020 -inch $(0.51$ $\mathrm{mm}$ ) inner diameter tubing, we could aspirate volumes up to approximately $15 \mu \mathrm{L}$ without contacting the valve. This feature enables the aspiration of reagents that may adversely affect the plastic components within the valve. Solvents such as acetonitrile, ethanol, methanol, and tetrahydrofuran have all been used successfully.

The solenoid driver box and the motion controller are mounted remotely. The cables connecting the driver box to the ink jets are routed, along with the fluid hoses. The X-Y substage was added to provide higher-resolution movements than those provided by the MICROLAB 2200. The MICROLAB 2200 steps in $100-\mu \mathrm{m}$ increments, while the substage allows for approximately $6-\mu \mathrm{m}$ steps. Such high resolution is unnecessary for most applications but aids in the construction of microarrays by allowing for a finer increment in stepping than that provided by the MICROLAB 2200. Ultimately, the density of the spots is limited by the precision of the MICROLAB 2200's positioning system. We found it to be reproducible to approximately $50 \mu \mathrm{m}$. Figure 2 shows 8 -inch slides that allow for mounting up to 24 standard microscope slides or two microplates. The left-hand side of the instrument is used for storing microplates, which house the samples, while target substrates, such as glass microscope slides, are mounted onto the substage. The substage is machined out of magnetic stainless steel, allowing for the use of magnets to secure the position of the target substrates.

The fluidic connections are illustrated in Figure 3. This diagram outlines the arrangement of the fluidic components and the flow directions for executing the steps of aspirating, dispensing, and washing for one channel. Aspiration proceeds the same as with an unmodified MICROLAB 2200, using the Eclipse's aspirate command. The only required additional step is to hold the ink-jet valve open before aspi-

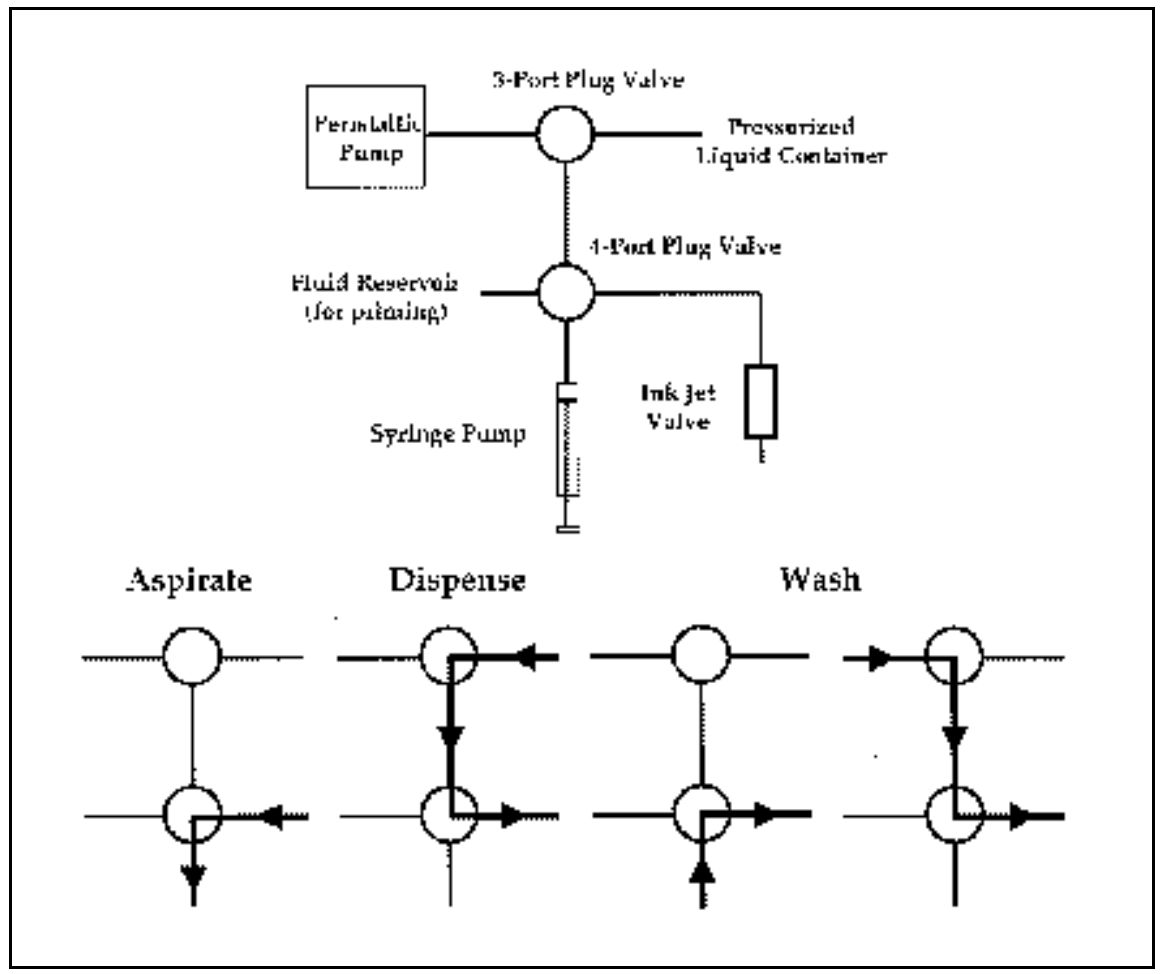

Figure 3. Diagram of the fluidic connections between various components of the liquid-handling system. The peristaltic pump and pressurized liquid container are connected to a three-port Hamilton MVP plug valve that connects to the top port of the syringe pump plug valve. The diagrams below describe the valve positions and the flow directions for the steps of aspirating, dispensing, and washing. ration. Using $10-\mu \mathrm{L}$ syringes, volumes as small as $10 \mathrm{~nL}$ can be aspirated (2). For low-volume dispensing, an air bubble between the sample and the system fluid prevents effective reagent jetting because the air bubble absorbs the pressure pulse. Therefore, an air gap is not used. This allows some mixing be- tween the sample and system fluid. We have found that aspirating a twofold greater volume than what is required is sufficient for preventing the delivery of a diluted sample. After aspiration, the ink-jet valve is closed and the plug valves are positioned to allow for a constant pressure head to be applied to the

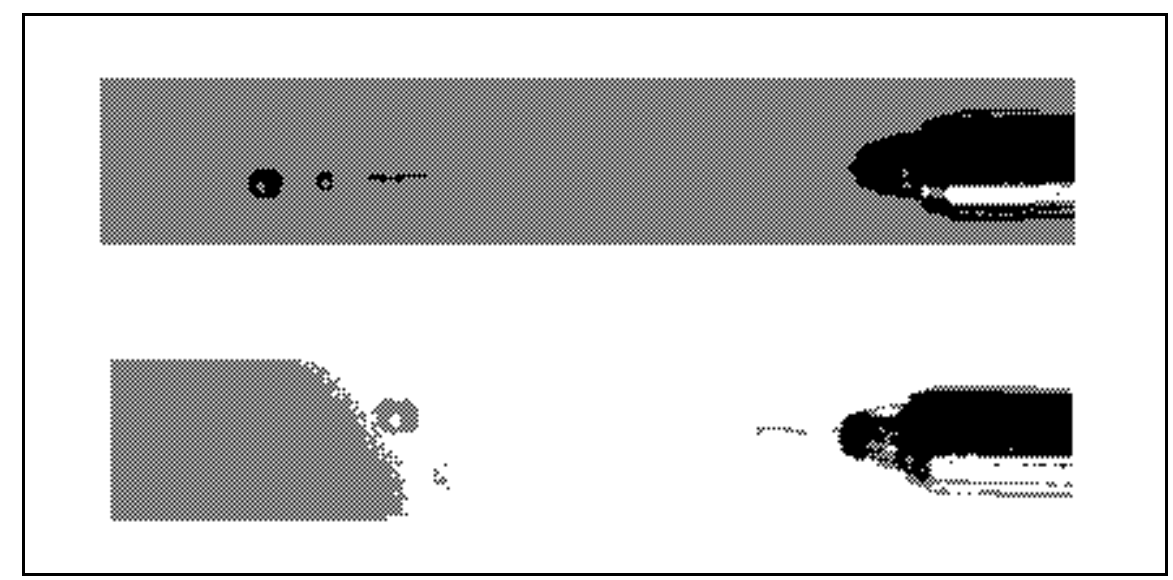

Figure 4. Images of liquid ejecting from the sapphire-tipped nozzle using either long (1 ms) or short $(\mathbf{0 . 2} \mathrm{ms})$ pulse widths. With long pulse widths, the liquid streams from the nozzle, eventually breaking up into individual droplets. With short pulse widths, it is possible to eject a single droplet. The images were taken using a microscope-mounted CCD camera and a handheld strobe to freeze the motion of the liquid. 


\section{Research Report}

ink-jet valves. The pressure regulator is typically set at $7.5 \mathrm{psi}$, but a range of pressures between 4 and 15 psi work suitably. The solenoid valve is rated for a maximum pressure of $10 \mathrm{psi}$, although no adverse effects were noticed when it was run at higher pressures.

For dispensing, the MICROLAB 2200 positions the sapphire tips above the substage. The executable software then coordinates the movement of the substage and the firing of the solenoid valves, while the MICROLAB 2200 holds the dispensing tips in a fixed position. When multiple tips are used, the stages can be programmed to move between the firing of successive tips to reduce the spacing between the dispensing events, thereby conserving the original sample order. This is especially useful when constructing small arrays compared to multi-tip, touch-off techniques in which the fixed-tip spacing limits the closeness of spot spacing. After dispensing, the MICROLAB 2200 positions the dispensing tips over the wash station. The solenoid valves are opened, the syringe barrels are emptied, and the dispensing tips are washed using the peristaltic pump, as diagrammed in Figure 3. Typically, a few hundred microliters of system fluid (water) is washed through the tips for 2 s. This is approximately 100 times the aspiration volume, which has been sufficient to prevent sample carryover. Optimization of the wash times or volumes has not been performed. Alternatively, the pressurized fluid source could be used to wash the tips.

Volume modulation is easily accom plished by altering the length of time the valve is open. As seen in Figure 4, this opening time is directly related to the volume of sample that is ejected. These images show that, under appropriate conditions, rapid actuation of the valve allows for the release of a single droplet. Longer actuation times cause the reagent to stream from the nozzle. As seen in Figure 5, the dispensed volume varies linearly with the valve opening time. Volumes greater than a few nanoliters can be reliably dispensed. The volume that is dispensed depends on multiple factors, including the applied pressure, the physical characteristics of the solution, the nozzle dimensions, and the valve opening time. The valve opening time is the simplest variable to modulate, and measuring the dispensed volume under a set of standard conditions provides an effective calibration. The lower limit of the valve actuation time also limits the minimum volume that can be dispensed. A small- er-diameter nozzle would aid in lowering the dispensed volume, although this would also lead to clogging and require slower aspiration.

The volumes displayed in Figure 5 were determined by weighing the total mass of multiple dispensing events. Typically, 500-1000 dispensing events were necessary to bring the weight into a measurable range of a typical analytical balance because the density of water is $1 \mu \mathrm{g} / \mathrm{nL}$. This calibration technique is simple to perform, although it is not informative with respect to the precision of individual dispensing events. For determining precision, we dispensed a concentrated solution of fluorescein into individual wells of a microplate and measured the fluorescence using a plate reader. Table 1 presents a summary of the results for four different pulse widths. In general, there were no missed deliveries, although the fluorescent signal began to tail off after a volume equivalent to approximately one-half of the aspirated volume was achieved. This decrease in signal is presumably because of the mixing of sam ple with the system fluid. Therefore, only dispensing events that correlate to approximately one-half of the aspirated volume were analyzed. The repeatability is as good or better than that reported

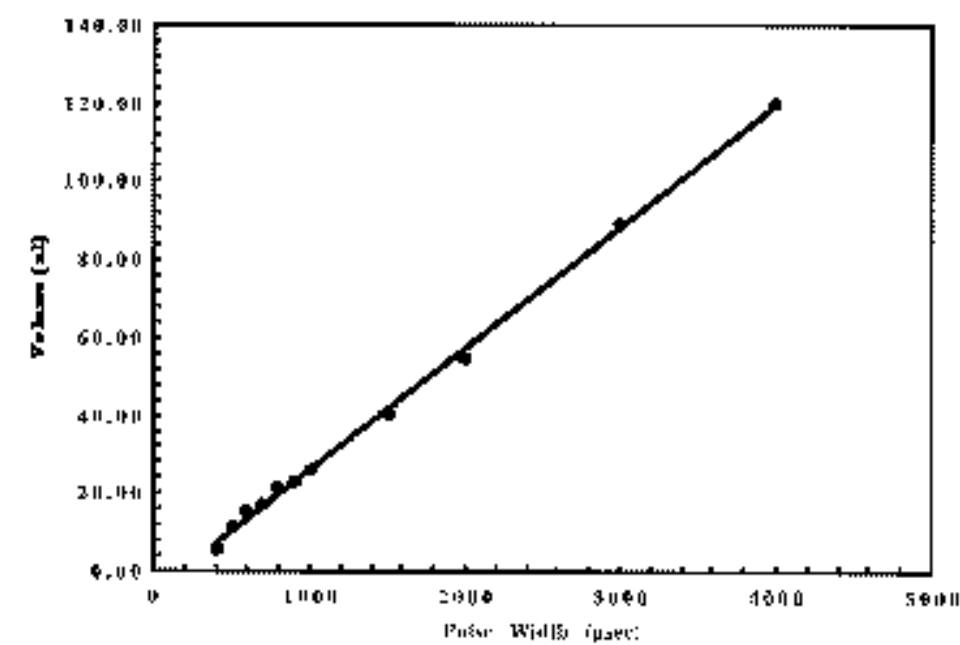

Figure 5. Graph of the volume ejected as a function of valve pulse width. A linear relation between the variables is observed, allowing the calibration of the volume dispensed under a particular set of conditions (i.e., pressure, pulse width, and nozzle dimensions). The volumes were determined by weighing the sum of 1000 dispensing events.

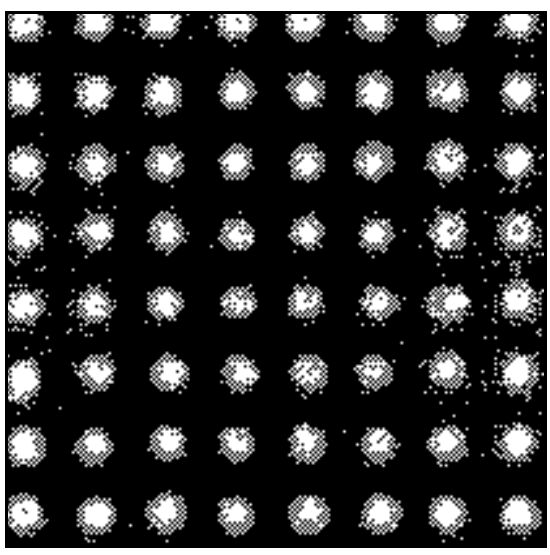

Figure 6. Image of a microarray created by the modified liquid-handling system. The array was created by the repeated dispensing of a Cy3labeled oligonucleotide onto a poly-L-lysinetreated microscope slide. The volume dispensed is approximately $3.5 \mathrm{~nL}$, and the spot pitch is 1 $\mathrm{mm}$. The reagent was dispensed from a height of $1 \mathrm{~mm}$ above the slide. The average spot diameter is $442 \mu \mathrm{m}$. The array was imaged and processed using a GeneTAC ${ }^{\mathrm{M}} 1000$ imager with Visage HDG analyzer software (Genomic Solutions, Ann Arbor, MI, USA). 
for similar low-volume dispensing (1), with a standard deviation of just a few nanoliters for average delivery volumes of 8.6-213.0 nL. Most variation is below $10 \%$, with the highest CV observed for the lowest-volume delivery. Table 1 also lists the volume determined from the weight of multiple dispensing events. These values are generally low er than those determined by the fluorescent technique, except for the lowestvolume delivery. There are several potential sources for the discrepancy and for the variability in the measurements. Slight evaporation may occur between collecting dispensing events and weighing, making the weight measurement technique less accurate. Additionally, the lowest-volume dispensing events are the most sensitive to compliance in the fluid lines. Slight air bubbles can adversely affect the delivery and the pressure drop across the nozzle. Care needs to be taken during aspiration to prevent the creation of air gaps. Withdrawing the syringe slowly and pausing briefly after aspiration ensures a complete sample collection.

Another testament to the repeatability of the dispensing events is demonstrated in Figure 6, which shows a fluorescence image of a Cy3-labeled DNA probe arrayed on a microscope slide. The $8 \times 8$ array was constructed by the repeated dispensing of a single reagent. No dispensing events were missed. The average spot diameter is $442 \mu \mathrm{m}$ with a standard deviation of $22 \mu \mathrm{m}$, or a CV of $5 \%$. These values were determined after calculating the spot area for each of the spots. The dispensing event volume was approximately $3.5 \mathrm{~nL}$. Similar arrays constructed using repeated aspiration, dispensing, and washing steps show similar results. The array density is lim ited primarily by the quantity of reagent that is dispensed and the contact angle between the fluid and the surface. Sam ple pitches of $400 \mu \mathrm{m}$ have been achieved on microscope slides. Lowering the limits of volume delivery is critical for increasing the array density.

\section{CONCLUSION}

We have developed a solenoidbased, reagent-jetting modification for extending the performance of a
MICROLAB 2200. The modifications allow for nanoliter-level dispensing and, with the addition of mechanical substages, improved spatial resolution of dispensing events. The instrument is capable of standard procedures, such as plate filling and sample transfer, and is capable of constructing DNA microarrays. The modifications can be extended to include multiple-dispensing tips for greater throughput. They should be adaptable to other systems or easily adapted for stand-alone operation. Most importantly, this approach to liquid dispensing enables the handling of nanoliter-scale volumes for the improved economy and throughput of biochemical assays.

\section{ACKNOWLEDGMENTS}

We acknowledge the engineering support of John Turner and Richard Hutchens from the Oak Ridge National Laboratory (ORNL). Additionally, we are grateful for the support and assistance of Jim Weber (Marshfield Medical Research Foundation), Peter Hoyt, Jim Johnson, Craig Storms, and the Hamilton Company. This research was sponsored by the Laboratory Directed Research and Development Program of ORNL, managed by UT-Battelle, LLC, for U.S. Department of Energy contract no. DE-AC05-00OR22725, and by National Institutes of Health grant no. R01 HL62681-02.

\section{REFERENCES}

1.Bateman, T.A., R.A. Ayers, and R.B. Greenway. 1999. An engineering evaluation of four fluid transfer devices for automated 384-well high throughput screening. Lab Robotics Automation 11:250-259.

2.Beattie, K.L., W.G. Beattie, L. Meng, S.L. Turner, R. Coral-Vazquez, D.D. Smith, P.M. McIntyre, and D.D. Dao. 1995. Advances in genosensor research. Clin. Chem. 41:700-706.

3.Blanchard, A.P., R.J. Kaiser, and L.E. Hood. 1996. High-density oligonucleotide arrays. Biosens. Bioelectron. 11:687-690.

4.Brandt, D.W. 1997. Multiplexed nanoliter transfers for high throughput drug screening using the Biomek 2000 and the high density replicating tool. J. Biomol. Screening 2:111116.

5.Cheung, V.G., M. Morley, F. Aguilar, A. Massimi, R. Kucherlapati, and G. Childs. 1998. Making and reading microarrays. Nat.
Genet. Suppl. 21:15-19.

6.Graves, D.J., H-J. Su, S.E. McKenzie, S. Surrey, and P. Fortina. 1998. System for preparing microhybridization arrays on glass slides. Anal. Chem. 70:5085-5092.

7.Knebel, G. 1998 High-throughput screening with 1536 wells. Am. Biotechnol. Lab. 3:22-24

8.Le, H.P. 1998. Progress and trends in ink-jet printing technology. J. Imaging Sci. Technol. 42:49-62.

9.Lemmo, A.V., J.T. Fisher, H.M. Geysen, and D.J. Rose. 1997. Characterization of an inkjet chemical microdispenser for combinatorial library synthesis. Anal. Chem. 69:543-551.

10.Marshall, A. and J. Hodgson. 1998. DNA Chips: An array of possibilities. Nat. Biotechnol. 16:27-31.

11.Okamoto, T., T. Suzuki, and N. Yamamoto. 2000. Microarray fabrication with covalent attachment of DNA using bubble jet technology. Nat. Biotechnol. 18:438-441.

12.Oldenburg, K.R., J-H. Zhang, T. Chen, A. Maffia III, K.F. Blom, A.P. Combs, and T.D.Y. Chung. 1998. Assay miniaturization for ultra-high throughput screening of combinatorial and discrete compound libraries: a 9600-well (0.2 microliter) assay system. J. Biomol. Screening 3:55-62.

13.Papen, R., K. Croker, and A. Kolb. 1998. Nanoliter dispensing technology. Genet. Eng. 18:16.

14. Ramsay, G. 1998. DNA chips: state-of-the art. Nat. Biotechnol. 16:40-44.

15. Roda, A., M. Guardigli, C. Russo, P. Pasini, and M. Baraldini. 2000. Protein microdeposition using a conventional ink-jet printer BioTechniques 28:492-496.

16.Stimpson, D.I., P.W. Cooley, S.M. Knepper, and D.B. Wallace. 1998. Parallel production of oligonucleotide arrays using membranes and reagent jet printing. BioTechniques 25:886-890.

Received 13 July 2000; accepted 27 October 2000.

Address correspondence to:

Dr. Mitchel J. Doktycz

Life Sciences Division

Oak Ridge National Laboratory

P.O. Box 2008

MS 6123

Oak Ridge, TN 37831, USA

e-mail:doktyczmj@ornl.gov 